\title{
Light and electron microscopy of globular hyaline inclusions in liver cells
}

\author{
J. C. E. UNDERWOOD \\ From the Department of Pathology, University of Sheffield
}

SYNOPSIS Numerous large globular hyaline inclusions were found in the liver cells of a patient with congestive cardiac failure due to cor pulmonale. The nature of the inclusions is discussed with particular emphasis on certain ultrastructural features which suggest a possible derivation from peroxisomes.

Large intracytoplasmic hyaline inclusions are unusual in biopsies of human liver and may cause diagnostic difficulty.

This paper describes a case in which numerous large $(15 \mu$ diameter) globular hyaline inclusions were a conspicuous feature of a needle liver biopsy. The appearances were studied by electron microscopy of biopsy and necropsy specimens. On the basis of the microscopic findings the nature of the inclusions is discussed.

\section{Case Summary}

The patient was aged 62 when first admitted to hospital with an acute exacerbation of chronic bronchitis. Respiratory disability had been present for 20 years; it had recently increased in severity and had been punctuated by several episodes of pneumonia. There was no relevant family history.

On clinical examination there was evidence of advanced chronic bronchitis with emphysema and cor pulmonale, complicated by severe congestive cardiac failure. The liver was found to be slightly enlarged and firm. There was no splenomegaly.

Relevant laboratory findings showed electrolytes to be normal. There was a slightly elevated blood urea $(50-70 \mathrm{mg} / 100 \mathrm{ml})$ throughout the illness. Total serum proteins were $6 \cdot 3 \mathrm{~g} / 100 \mathrm{ml}$ (albumin $3.9 \mathrm{~g} / 100 \mathrm{ml}$ ). Electrophoresis was normal. Serum bilirubin was $2.8 \mathrm{mg} / 100 \mathrm{ml}$ (positive direct van den Bergh), SGOT $45 \mathrm{mIU}$, SGPT $24 \mathrm{mIU}$, alkaline phosphatase 42 King units, slight excess of urinary urobilinogen. A bromsulphthalein test gave $14 \%$ retention at 45 minutes, $10 \%$ retention at 60 minutes.

There was good clinical improvement in cardioReceived for publication 9 June 1972. respiratory function in response to treatment with tetracycline, digoxin, choline theophyllinate, frusemide, and spironolactone with potassium supplement. However, as the hepatomegaly persisted a percutaneous needle liver biopsy was done two weeks after admission. The histological findings are discussed below.

After discharge from hospital he remained relatively well, apart from a further episode of acute bronchitis, but the liver remained large. Ten months later there was a sudden and severe deterioration in the patient's condition with marked dyspnoea, jaundice, ascites, and peripheral oedema. He failed to respond to treatment and died two days after admission.

\section{NECROPSY}

The relevant findings were confined to the cardiorespiratory system and liver. The lungs exhibited chronic bronchitis with severe emphysema. There was marked right ventricular hypertrophy. The liver weighed $1740 \mathrm{~g}$ and although firm the capsular surface was smooth. The cut surface displayed a fatty texture and appeared congested. The bile ducts were normal but the gallbladder enclosed a few mixed stones. The hepatic and portal veins were normal. Samples of liver were taken for electron microscopy and routine histology was carried out on all organs.

\section{Methods}

The liver biopsy was received in formol-saline and, after routine processing, paraffin sections were cut. It was decided to attempt electron microscopy of the tissue in order to elucidate the unusual appearances 
seen in paraffin sections. The biopsy tissue was removed from wax after gentle heating and washed ovcrnight in xylol. After rehydration by passage through graded alcohols the sample was postfixed in $2 \%$ osmium tetroxide and embedded in Araldite. Thin sections were stained with lead citrate for electron microscopy. It was not possible to obtain a further sample of liver during life.

Necropsy was carried out 10 hours after death. A thin slice of liver was fixed in cold neutral phosphatebuffered $3 \%$ glutaraldehyde, washed in buffer, postfixed in $2 \%$ osmium tetroxide, and embedded in Araldite. Lead citrate-stained thin sections were examined by electron microscopy.

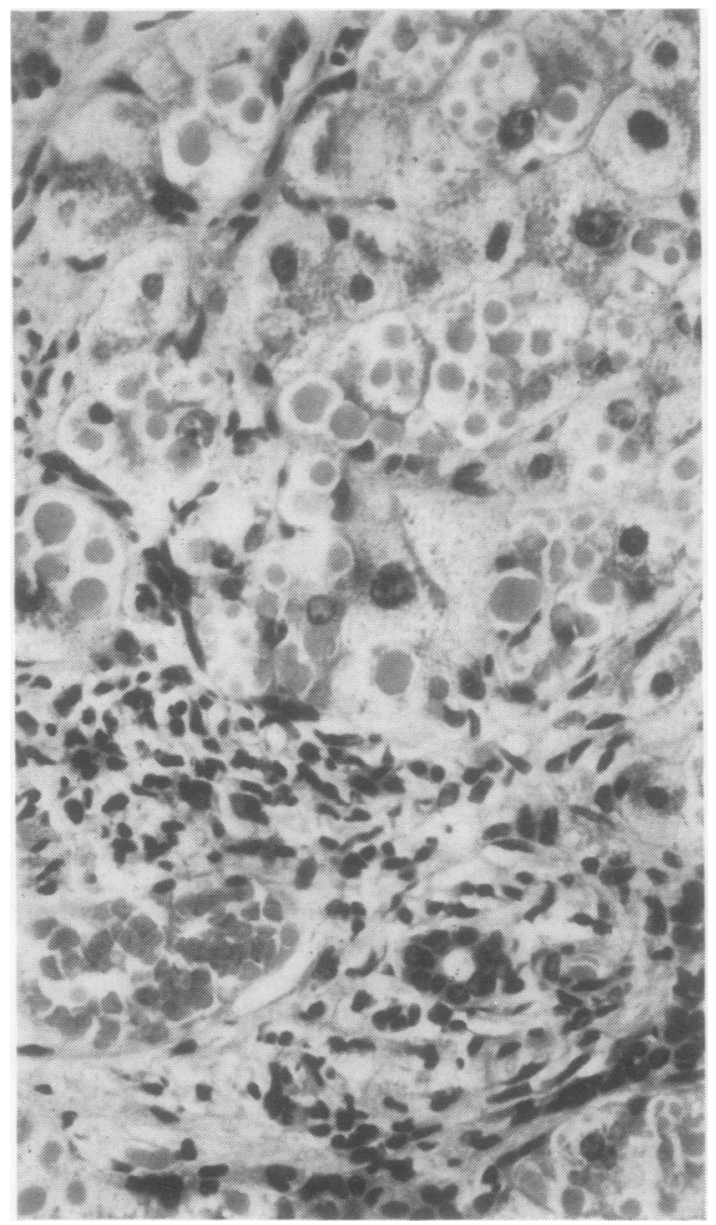

Fig. 1.
The paraffin sections of formalin-fixed liver were stained with haematoxylin andeosin, phosphotungstic $\underline{\underline{\sigma}}$ acid haematoxylin (PTAH), phloxine-tartrazine, and periodic-acid Schiff (PAS) before and after diastase $\stackrel{9}{9}$ treatment. A frozen section was stained with Oil $\overline{0}$ Red $\mathbf{O}$.

\section{Results}

LIGHT MICROSCOPY

The liver biopsy was fragmented. Cirrhosis was not $\vec{\circ}$ evident but there was definite irregular expansion of $\overrightarrow{\vec{\omega}}$ portal tracts by fibrous tissue in which was a $\stackrel{\omega}{\stackrel{D}{~}}$ moderate infiltrate of lymphoid cells. The bile ducts $\frac{}{8}$

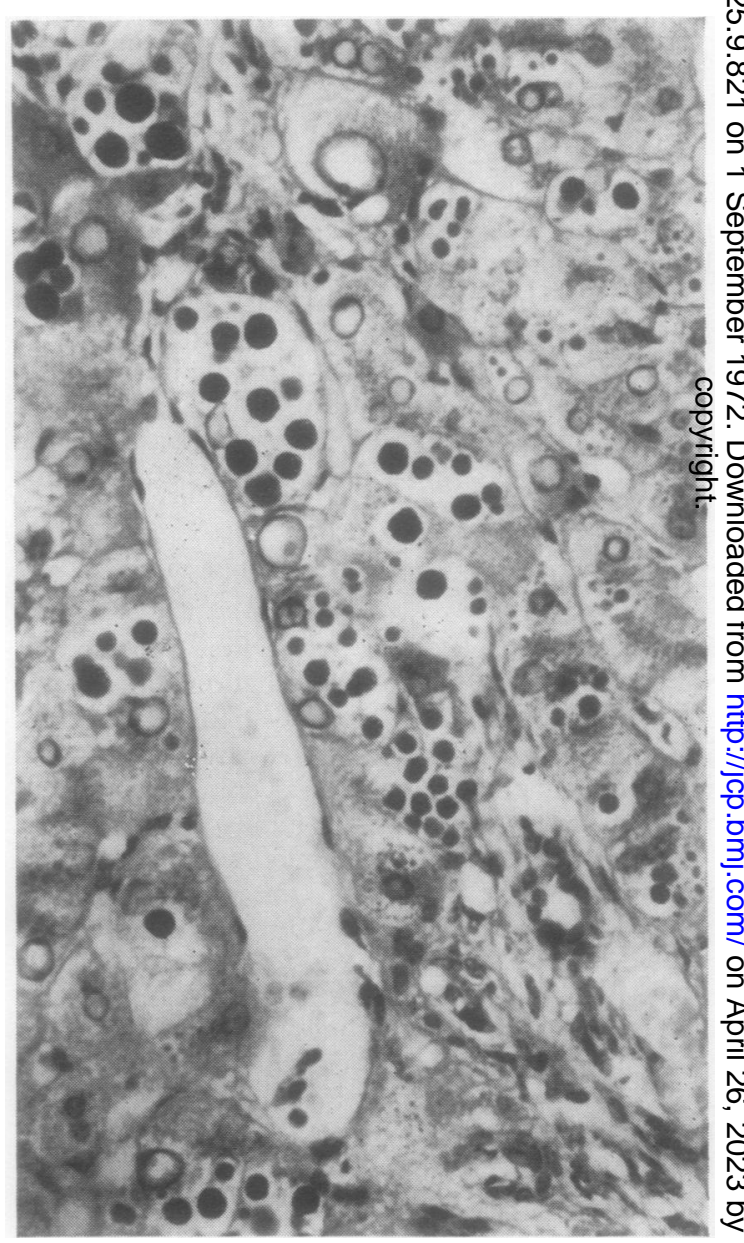

Fig. 2.

Fig. 1 Liver biopsy. Numerous globular hyaline inclusions in cytoplasm of peripheral hepatocytes. Portal tract shows irregular expansion by fibrous tissue bearing a lymphoid infiltrate (haematoxylin and eosin $\times 250$ ).

Fig. 2 Liver biopsy. Strongly periodic acid-Schiff $(P A S)$ positive inclusions after diastase treatment. Some liver cells show nuclear vacuoles $(P A S$ after diastase $\times 250)$. 


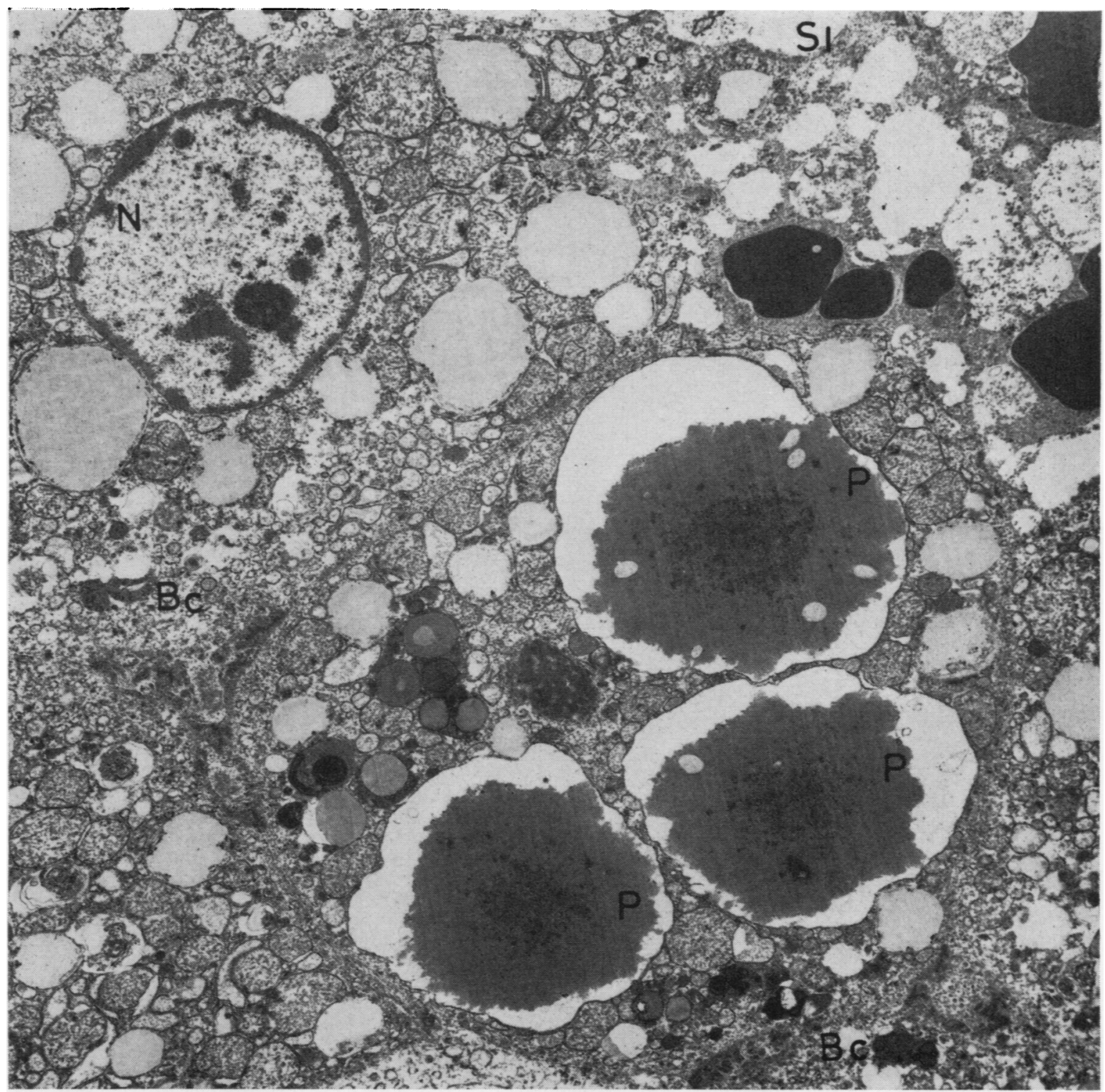

Fig. 3 Postmortem liver. Three large inclusions $(\mathrm{P})$ in cytoplasm of a hepatocyte. The central dense core is shown and each inclusion is limited by a single membrane. Nucleus $(\mathrm{N})$ in adjacent liver cell, bile canaliculi $(\mathrm{Bc})$ and the edge of a vascular sinusoid $(\mathrm{Si})$ containing red cells are also present $(\times 5000)$.

and radicles of the hepatic artery and portal vein were normal but the limiting plate of liver cells was poorly defined. No obvious features of passive venous congestion were found. The most striking abnormality was the presence of numerous eosinophilic globular hyaline inclusions within the cytoplasm of liver cells, predominantly in periportal zones (Fig. 1). These inclusions measured up to $15 \mu$ in diameter and the average size was approximately $5 \mu$. Some cells enclosed only two or three large inclusions in the plane of section whereas others were packed with many small globules. The inclusions were very strongly PAS positive (Fig. 2) and unaffected by diastase. Sections lightly stained with 


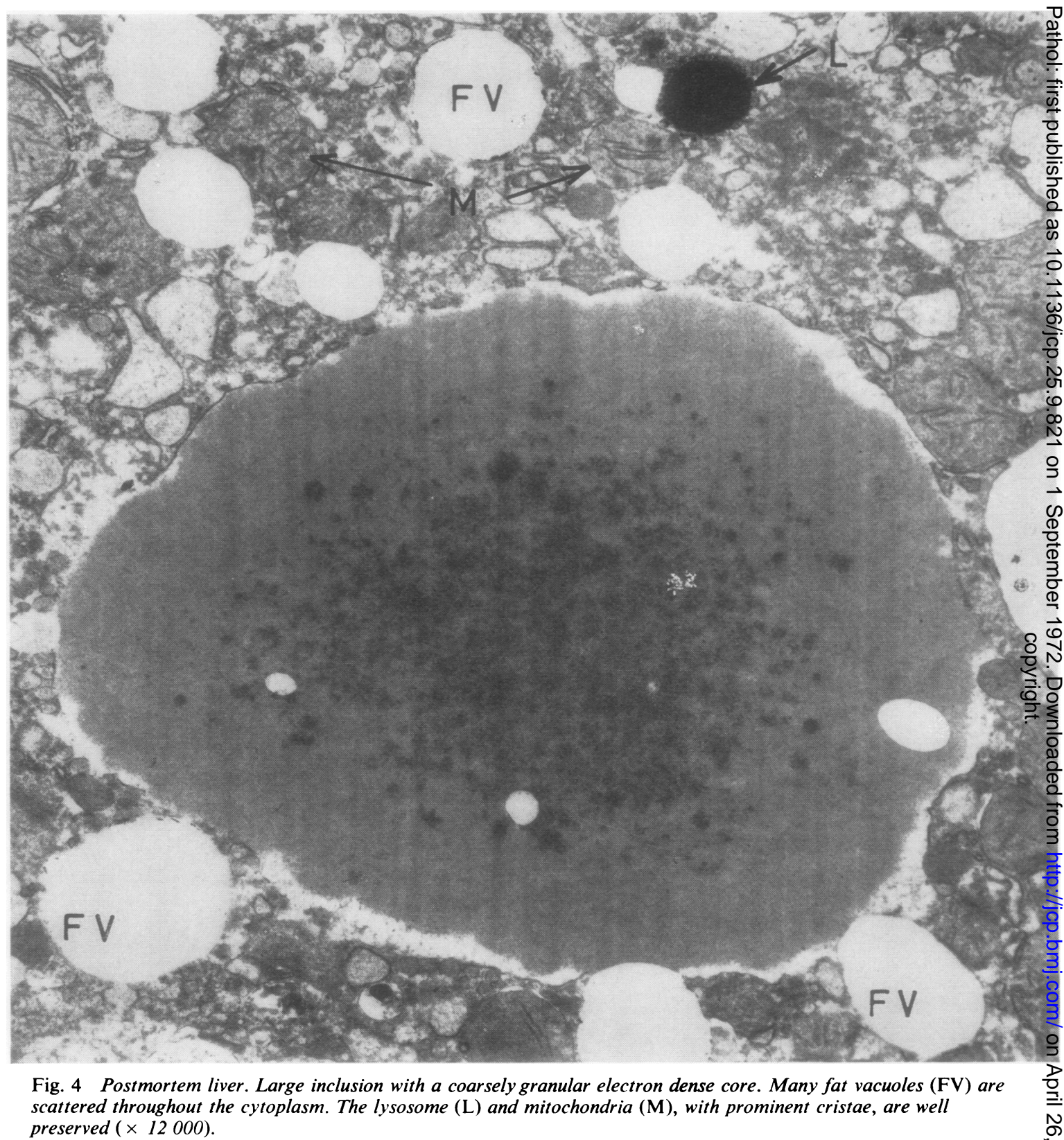

(L) and mitochondria $(\mathrm{M})$, with prominent cristae, are well preserved $(\times 12000)$.

PTAH showed these inclusions to possess a dense centre and a weakly staining periphery in contrast to the uniform PAS reaction. The inclusions were phloxine positive, but no stainable lipid was detected.

Globular hyaline inclusions were also numerous in the samples of liver removed at necropsy and gave similar staining reactions. There was, however, more disruption of hepatic architecture by portal fibrous tissue but there was no established cirrhosis. More obvious features of passive venous congestion were present with much fatty change. No inclusions were found in any other organ examined.

\section{ELECTRON MICROSCOPY}

The degree of preservation of the necropsy samples was surprisingly good and fine intracellular detail 


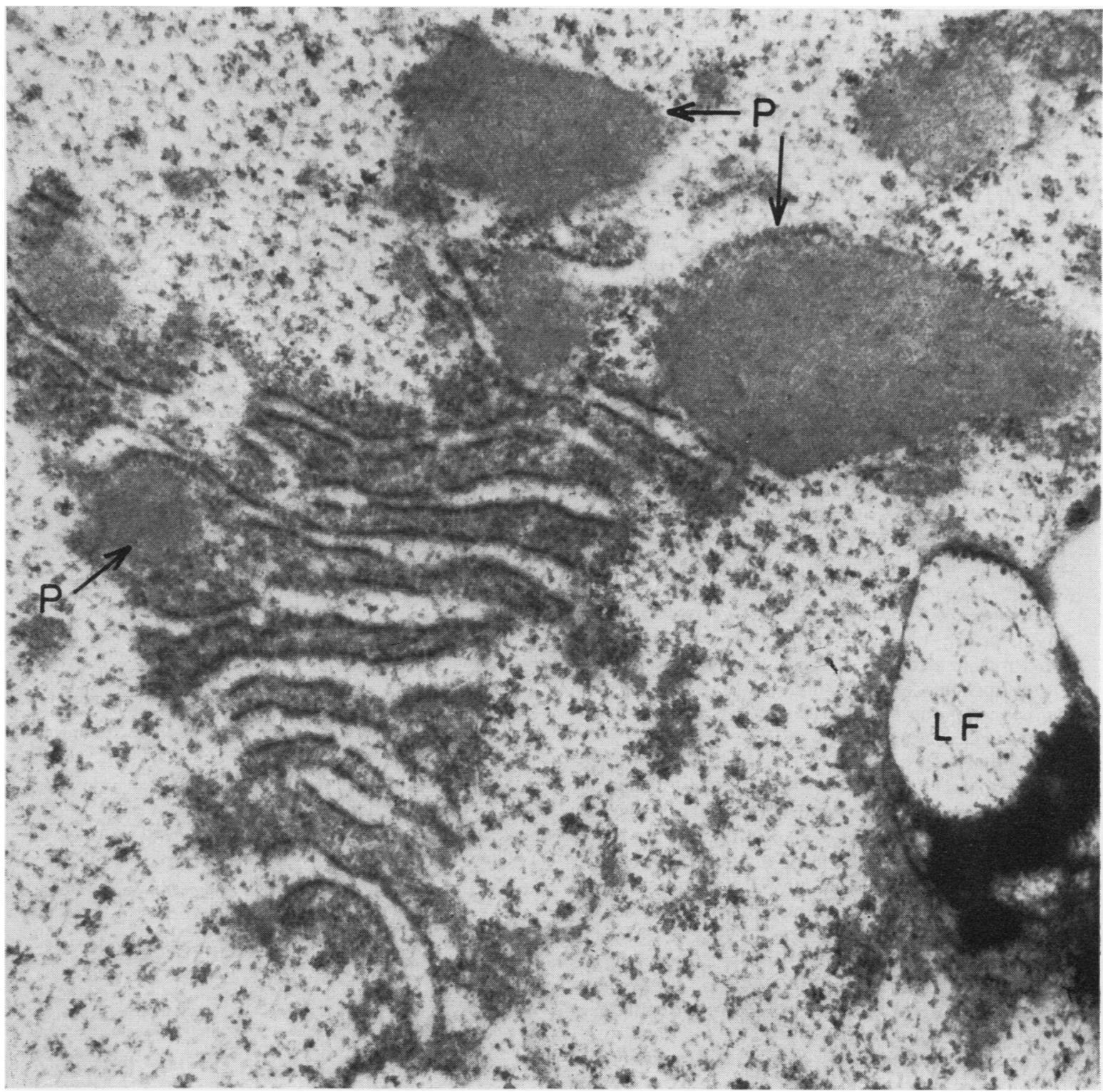

Fig. 5 Liver biopsy. Cisternae of endoplasmic reticulum filled with flocculent material of medium electron density. Several large intracisternal aggregates may be peroxisomes $(\mathrm{P})$. A lipofuscin $(\mathrm{FL})$ granule is also present $(\times 40000)$.

was observed in the biopsy.

The globular cytoplasmic inclusions had a characteristic structure, consisting of a fine granular matrix of medium electron density with a central electron-dense coarse granular core (Figs. 3 and 4). Each inclusion was enclosed by a clearly defined single membrane, often separated from the inclusion by a narrow space. In the biopsy smaller inclusions were found in Golgi cisternae (Fig. 5) and there were transitional forms between these and the larger bodies.

Mitrochondria were well preserved with clearly visible cristae and no abnormal forms were seen.

\section{Discussion}

Mallory (1901), in an early account of hepatic necrosis, drew attention to the occasional presence 
of cytoplasmic inclusions in liver cells. He described one type as thread-like and subsequently regarded this as a feature of liver disease due to alcoholism. These inclusions are now referred to as 'alcoholic' or reticular hyaline. A second type of inclusion was described as hyaline, globular, and eosinophilic, sometimes found within a small vacuole. These globular inclusions were found by Mallory predominantly in centrilobular cells.

In human material globular hyaline inclusions are most often seen in malignant hepatomas (Ishak and Glunz, 1967; Norkin and Campagna-Pinto, 1968). However, Pappenheimer and Hawthorne (1936) recorded the presence of similar inclusions in over $30 \%$ of cases of liver disease. The inclusions were small (less than $5 \mu$ in diameter), showed no correlation with specific diseases, and, in contrast to the findings of others, were not found in hepatomas. It is probable that the inclusions these authors reported are of a different nature to those in the present case. In their series they were usually found in centrilobular hepatocytes. Indeed globular hyaline inclusions in liver cell cytoplasm may be a heterogenous group sharing only light microscopic appearances in common. Steiner, Jézéquel, Phillips, Miyai, and Arakawa (1965) reviewed the fine structure of various liver cell inclusions and showed a wide spectrum of morphological types. However, inclusions of the type portrayed in this case do not appear to have been previously described.

Globular inclusions in the liver cells of patients with emphysema due to $\alpha_{1}$ antitrypsin deficiency have been recently reported (Lieberman, Mittman, and Gordon, 1972). These authors describe PASpositive inclusions up to $20 \mu$ in diameter of moderate electron density and bound by a single membrane. One marked difference is the absence of a central dense core, a striking feature in the present case. Unfortunately it was not possible to exclude $\alpha_{1}$ antitrypsin deficiency in this case, and, although on balance this seems unlikely, the disorder remains a remote possibility.

The staining characteristics suggest that these inclusions are largely glycoprotein, and the smaller forms bear some ultrastructural resemblance to peroxisomes or microbodies (de Duve, 1969). These organelles are normally $0 \cdot 4-0 \cdot 8 \mu$ in diameter in $D^{0}$ human liver and possess a well defined single $\overrightarrow{\vec{\sigma}}$ membrane. They are often closely related to endo- $\frac{\text { o }}{-}$ plasmic reticulum (Novikoff and Shin, 1964) and $\underset{\vec{\rho}}{\vec{\rho}}$ this was a prominent feature of the smaller inclusions in this case. Ma and Biempica (1971) have shown that human hepatic peroxisomes occasionally have a granular dense core or nucleoid although this is an unusual feature. Peroxisomes are also found in $\triangle$ mammalian renal tubular epithelial cells and in vitro क have peroxidase (catalase) activity. Lavin and Koss $\overrightarrow{0}$ (1971) have shown, in an experimental study, that renal tubular peroxisomes in rats can attain a $\vec{\omega}$ diameter of $4 \mu$ following a single dose of cyclophosphamide. However, it seems unlikely that the patient's therapy could have been responsible for $\mathcal{N}$ these appearances and the pathogenesis remains obscure.

I am grateful to $\operatorname{Dr}$ A. Kennedy for advice and encouragement and to Dr A. W. D. Leishman for $\mathbb{D}$ his permission to publish details of this case. $\mathrm{Mr}$ T. E. Durrant prepared the specimens for electron microscopy.

References

de Duve, C. (1969). The peroxisome: a new cytoplasmic organelle Proc. Roy. Soc. B., 173, 71-83.

Ishak, K. G., and Glunz, P. R. (1967). Hepatoblastoma and hepat $\sigma^{+}$ carcinoma in infancy and childhood. Cancer (Philad.), 20, 396-422.

Lavin, P., and Koss, L. G. (1971). Effects of a single dose of cyclophosphamide on various organs in the rat. II. Electron microscopic study of the renal tubules. Amer. J. Path., 62, 169-180.

Lieberman, J., Mittman, C., and Gordon, H. W. (1972). Alpha antitrypsin in the livers of patients with emphysema. Science, 175, 63-65.

Ma, M. H., and Biempica, L. (1971). The normal human liver cell: cytochemical and ultrastructural studies. Amer. J. Path., 62, 353-390.

Mallory, F. B. (19J1). Necrosis of the liver. J. med. Res., 6, 264-280.

Norkin, S. A., and Campagna-Pinto, D. (1968). Cytoplasmic hyaline inclusions in hepatoma. Arch. Path., 86, 25-32.

Novikoff, A. B., and Shin, W. Y. (1964). The endoplasmic reticulum in the Golgi zone and its relation to microbodies, Golgi apparatus and autophagic vacuoles in rat liver cells. J. Microscopie, 3, 187-206.

Pappenheimer, A. M., and Ha:t thorne, J. J. (1936). Certain cytoplasmic inclusions of liver cells. Amer. J. Path., 12, 625-633.

Steiner, J. W., Jézéquel, A. M., Phillips, M. J., Miyai, K., and D Arakawa, K. (1965). Some aspects of the ultrastructural pathology of the liver. Progr. liver Dis., 2, 303-372. 